the volume by omitting altogether those sections dealing with endocrine and "trophic" disorders. In this way space has been found for a great deal of new material. The text-book can now be considered "up-to-date" and it includes references to electro-encephalography, the neuro-avitaminoses, and the neutropic virus affections. Dr. Russell Brain has also added an important and very readable chapter on the psychological manifestations of organic nervous disease.

This is a very excellent text-book of neurology, and indeed for the graduate studying for higher degrees, as well as for the brighter medical student, this book can confidently be recommended as the most satisfactory investment at present available.

\section{AN INTRODUCTION TO BIOCHEMISTRY}

\section{(Second Edition).}

By W. R. Fearon, M.A., Sc.D., M.B., F.I.C. William Heinemann Medical Books, Ltd. London. 1940. Price 17s. 6d.

Professor Fearon as he says in his preface, has approached his subject from the unusual path of inorganic biochemistry. In pursuance of this aim Part I of the book contains an extensive and informative chapter devoted to a consideration of not only the more common but also of those rarer elements concerned in the phenomenon of life; incidentally this chapter contains material from the Transactions of the U.S.S.R. Academy of Science not hitherto available in the English language. The remainder of Part $I$ is concerned with inorganic compounds, and such principles of physical chemistry as the Ionic Theory, Donnan Equilibrium, and Colloids without a thorough understanding of which the nature of the fundamental differences between the body and the test tube cannot be grasped.

An admirable compromise has been struck between facts and theories, and whilst the book is altogether exceptional in the quantity of very recent work incorporated, the author has kept in mind the important fact that the book is being written not for biochemists, but for less knowledgeable persons who require a simplified and more dogmatic presentation of the subject.

It is a pity that Professor Fearon has not mentioned in his chapters on Hormones that while the traditional view is that the parathyroid hormone controls the blood calcium level, a growing body of opinion considers that this effect is secondary, and that the primary effect of the hormone is upon the blood inorganic phosphate level; of particular interest in this connection is the recent paper of Helfet (Brit. Jr. of Surg., April, 1940) in which the implications of this latter theory are applied clinically with promising results.

Perhaps a rather greater degree of familiarity with the fundamentals of organic chemistry has been assumed for readers of this book than is usually the case with this type of publication, though certainly no excessive assumptions have been made on these grounds. The space saved by the omission of this elementary organic chemistry has been employed in the expansion of the chapters on such subjects of rapidly increasing importance as Tissue Respiration.

The average medical student will probably find this book a little long and a trifle too detailed for his needs as it contains considerably more material than that usually demanded for examination purposes, at the same time the reader of this book will acquire quite a fair knowledge of pure physiology.

While not a practical manual this book contains instructions for practical work to illustrate the text, this is of course admirable as a means of bringing practice and theory into proper proportion. A short appendix has been included, which lists the composition and uses of the chief biochemical reagents.

The author's style is both clear and concise, and care has been taken to prune the text of ambiguous or misleading statements in the avoidance of which pitfalls the author has been uniformly successful.

This is a book which if not one of the most simple is most certainly one of the best introductions to the subject yet written, and is to be recommended to all who wish to obtain an insight into the trend of modern biochemistry.

\section{ELEMENTARY PATHOLOGICAL HISTOLOGY.}

By W. G. Barnard, F.R.C.P. (2nd Edition). Crown 4to. H. K. Lewis \& Co., Ltd. 1940. Price 10s. 0d. net.

This book, which is of the nature of an atlas of morbid histology, contains 181 illustrations of which 8 are in colour. In view of the expense of producing these, the book is an object lesson on what can be done for the modest sum of ten shillings.

The author has had the courage to do what the author of this review has always longed to be able to do-to produce a text-book on morbid histology, illustrated largely by photomicrographs. In other contemporary works on this subject (notably that by the late Prof. Donaldson), the authors have sought to make things easier for the student by utilising only careful drawings, mostly imitations of photomicrographs, at the production of which the late Mr. Richard Muir so greatly excelled. While these are admirably lucid, they have the disadvantage that they do not really resemble what the student sees through the microscope. The illustrations in this book consist largely (though not entirely) of excellent photomicrographs which, though in monochrome, really do show the picture seen through the microscope.

The author is to be congratulated on these photomicrographs, which are of a high order of excellence. Almost anyone can get a good photomicrograph of such tissues as thyroid, but it takes real skill to produce clear crisp pictures of, for example, the edge of a gumma. The flatness of field of the larger photomicrographs is surprising to those who know how difficult it is to attain. 